\title{
Gravitational waves and neutrinos joint searches in the Mediterranean Sea
}

\author{
Bruny Baret ${ }^{1, *}$ on behalf of the ANTARES and KM3NeT collaborations \\ ${ }^{1}$ Laboratoire Astroparticule et Cosmologie, 10 rue Alice Domon et Léonie Duquet, 75013 Paris
}

\begin{abstract}
The recent detection of gravitational waves (GW) have launched effectively the field of multimessenger astronomy. High energy neutrinos (HEN) have been detected as a diffuse flux and show indications of transient point sources. They can bring crucial informations on cataclysmic cosmic events by identifying hadrons acceleration to high energies and pointing back to the source at the degree level which is an asset for follow-up of GW events with photonic telescopes. We will review the current searches for joint sources of gravitational waves and high energy neutrinos performed with the ANTARES telescope and the perspectives offered by its successor KM3NeT.
\end{abstract}

\section{Introduction}

The impressive follow-up campaign [1] triggered by the detection of gravitational waves and gamma rays from the merger of two neutrons stars has shown the potential of the multimessenger approach in the study of transient high energy cosmic events. High energy cosmic neutrinos can bring crucial information in this context, tracing the presence of accelerated high energy hadrons in this kind of events and enabling in case of detection a faster and most efficient follow-up thanks to their degree scale pointing and all-sky monitoring. In addition, joint searches for sub-threshold coincident events with gravitational interferometers and high energy neutrinos would also help characterize the population of sources than could fill the gap between core collapse supernovae and gamma ray bursts [2]. We will review here such searches that have been performed with the ANTARES neutrino telescope and the perspectives for its successor KM3NeT.

\section{Gravitational wave alerts follow-ups}

Since the first detection of GW from a binary black hole (BBH) merger by the LIGO observatory, the ANTARES neutrino telescope has participated in the global follow-up efforts [5] in collaboration with the IceCube neutrino observatory. The first phase of such searches relies on the $2 \pi$ steradian continuous monitoring of the sky by neutrino telescopes in the muonic channel used at this stage due to its sub-degree angular resolution. Thanks to an online stream of fast event reconstruction a notice can be sent to the GCN network within less than a day stating the detection or not of a coincident high energy neutrino within a $\pm 500 s$ time interval [3]. Such a detection would reduce the search error box of further follow-ups by pointing

\footnotetext{
*e-mail: baret@in2p3.fr
} 
telescopes from 10-100 to 1 square degrees sparing useless scans and hence allowing a faster characterization of the source of which the luminosity is typically decreasing as power laws with time.

A refined search is then performed during the following weeks using refined calibrations and reconstructions in order to provide high energy neutrino fluence limits within the GW error box. This processing and the use of relaxed data quality cuts aiming at a fortuitous coincidence probability of $2.7 \times 10^{-3}$ increase the probed volume of the Universe by a factor 1.5 to 2 with respect to quick searches performed with typical point source searches quality cuts. This makes ANTARES the most sensitive neutrino telescope to the southern sky between $100 \mathrm{GeV}$ and $100 \mathrm{TeV}$, the higher part of the spectrum being dominated by IceCube [5]. After the search using up-going muon neutrinos only for the follow-up of the first BBH, each subsequent alert was investigated with improved data-analyses techniques like the inclusion of downward going muon neutrinos to cover the full GW error box and systematic uncertainties for the BBH event GW70104 [7] also presented in Fig. 1. For the follow-up of the binary
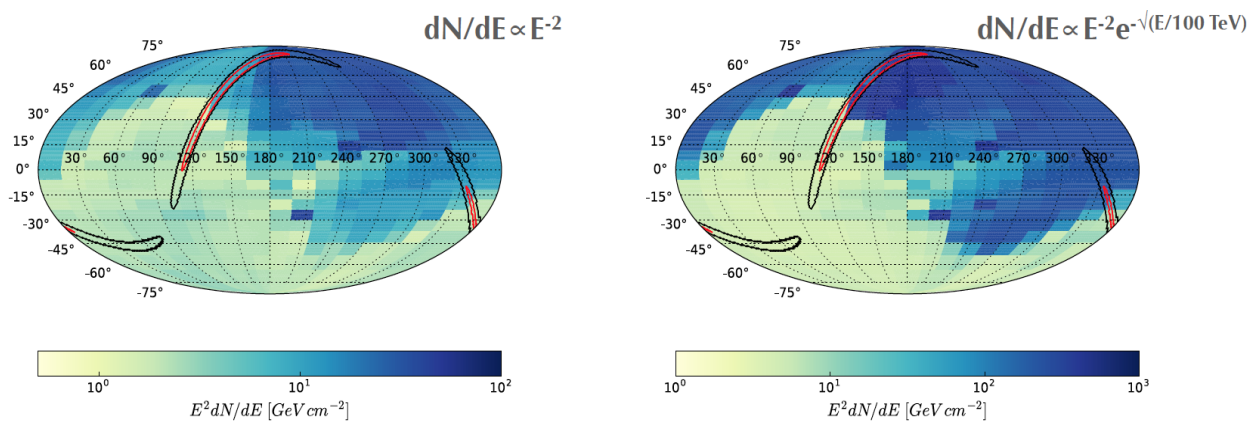

Figure 1. High energy neutrino fluence upper limits sky maps in equatorial coordinates at the time of GW170104 for two source spectra hypotheses [7]. The black and red contours delimit the 50 and 90 percent confidence level GW error boxes.

neutron star event GW170817 the so called cascade channel including upward going events was included making it an all-flavor search [1]. Despite the absence of a detection the limits constrained the viewing angle of the jet axis of the short gamma ray burst to be above $5^{\circ}$ for most optimistic models as seen in Fig. 2, in agreement with electromagnetic observations.

\section{Sub-threshold symmetric joint searches}

On top of the search for HEN counter part emission of strong GW events, the search for coincident sub-threshold events, obtained by relaxing data selection quality cuts, can even stronger constrain the joint source population. The idea is to search for excesses taking into account the directional overlap, the expected energy spectrum for the background neutrino hypothesis and the GW signal to noise ratio as in [4]. One proposed approach is to apply quality cuts on neutrino and gravitational wave data to maximize the number $\mathcal{N}_{\text {GWHEN }}$ of detectable sources emitting both GW and HEN while keeping the instantaneous false alarm probability constant at $2.7 \times 10^{-3}$. Let us assume that the sources are all identical and radiate $E_{G W}$ in GW and emit a fluence $\varphi_{v}$ in HEN. Let us also assume that their population is isotropic so their density per unit time and volume $R$ is a constant. The number of detectable sources 

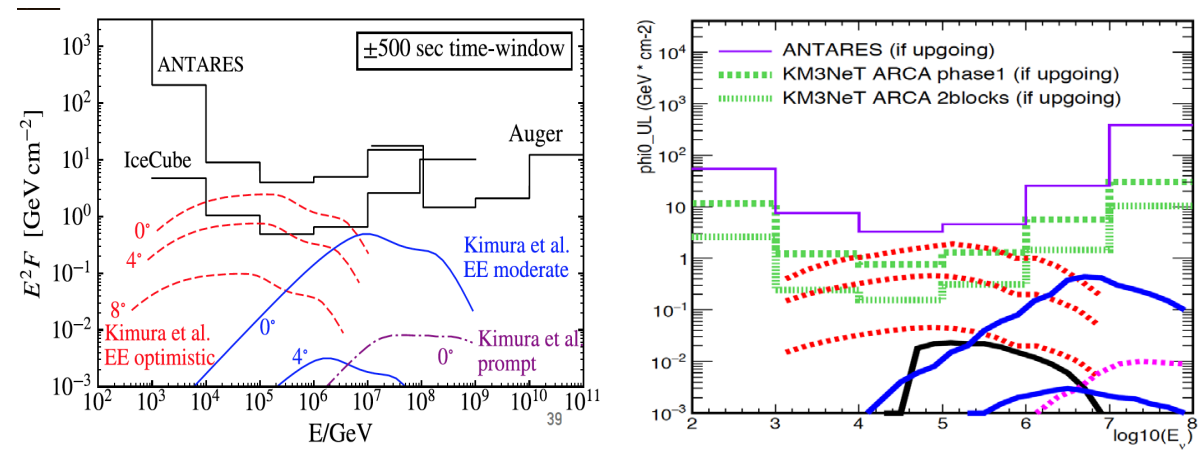

Figure 2. Left: High energy neutrino fluence upper limits for the ANTARES, IceCube and AUGER detectors in the direction of GW170817 together with model predictions [9]. Right: Same as left for ANTARES and KM3NeT for its different construction phases in case the source was in the up-going events field of view. The Baikal GVD experiment has performed a similar analysis, see [8].

is

$$
\mathcal{N}_{\text {GWHEN }}(\text { cuts })=\iint d t d^{3} \Omega \mathcal{R}(r, t) \epsilon_{\nu}(\text { cuts }) \epsilon_{G W}\left(\text { cuts } ; E_{G W}, r\right)
$$

where $\mathcal{R}(r, t)=R \mathcal{P}\left(N_{v}>0 \mid \varphi_{v} /\left(4 \pi r^{2}\right)\right)$ is the density of detectable sources with $r$ and $t$ the sources distance and time of detection and $R$ the population density. Following [12] one obtains:

$$
\mathcal{N}_{\text {GWHEN }}\left(\Lambda, \rho_{\text {threshold }}\right) \propto \epsilon_{v}(\Lambda) / \rho_{\text {threshold }}
$$

with $\Lambda$ and $\rho_{\text {threshold }}$ being respectively the track quality of reconstructed HEN tracks and the $\mathrm{GW}$ interferometer signal to noise ratio cut values. Applying this selection scheme makes takes advantage of the low coincident background to enhance the detector sensitivity. The obtained population constrains as estimated for S6 run LIGO data [12] is compared on Fig. 3 with the search performed by IceCube [11]. Another option is to optimize the HEN selection cuts in a model independent way inspired by the selection applied for follow-up searches. These time dependent cuts are defined such that the probability of having a reconstructed event in a regions of the sky drawn from the distribution of expected GW events in a $\pm 500 \mathrm{~s}$ window corresponds to a significance at the $3 \sigma$ level. This selection scheme has been used for the search of coincident events with Ligo O1 run together with IceCube [13], leading to the population constraints presented on Fig. 3 right.

\section{Perspectives}

The KM3NeT observatory is currently under construction as successor of ANTARES and will increase the sensitivity of future searches to the level of IceCube and beyond. The combination of sub-degree angular resolution, lower muon background and high effective area will make KM3NeT a powerful tool for the follow-up of future promising GW events. Fig. 2 right shows the sensitivity that one can expect with an event equivalent to GW170817. Current limits are barely reaching the parameter space of known objects like core collapse supernovae or binary neutron stars (see Fig. 3) whereby the addition of a complementary neutrino detector of the size of IceCube will push the sensitivity into the physically interesting region. 

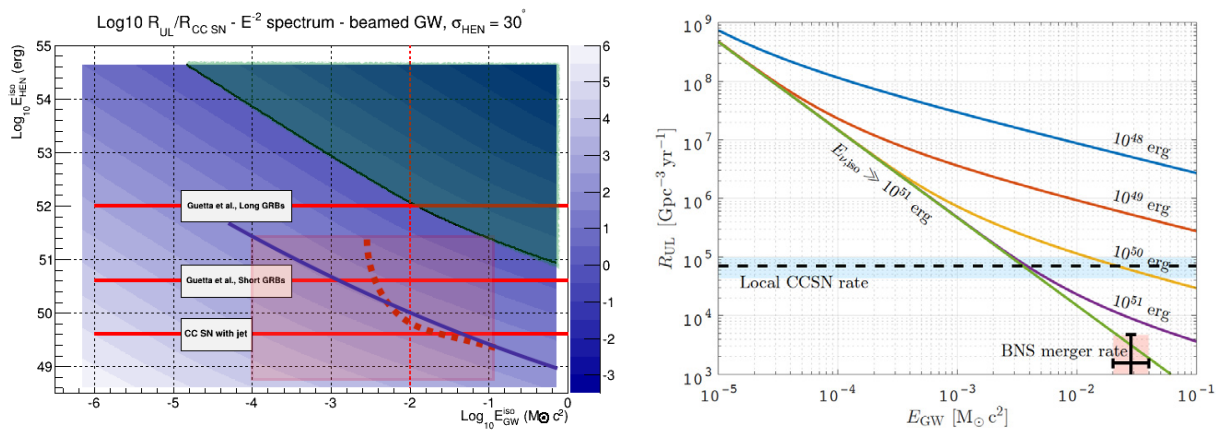

Figure 3. Left:Estimated upper limits (color scale) for the rate density of GW+HEN sources as functions of emitted energy in GW and isotropic energy emitted in neutrinos during S6 Ligo observations [12]. The dotted red line is the equivalent of the thick blue line from IceCube search [11] which was limited to the red shaded area. Right: Upper limits for the rate density of GW+HEN sources as functions of emitted energy in $\mathrm{GW}$, for different values of the isotropic energy emitted in neutrinos during O1 Ligo observations [13].

\section{References}

[1] B. P. Abbott et al., ApJL 12848 (2017)

[2] Shin'ichiro Ando et al., Rev.Mod.Phys. 85, no.4, 1401 (2013)

[3] B. Baret al., Astropart.Phys. 351 (2011)

[4] B. Baret et al., Phys.Rev. D85 103004 (2012)

[5] Adrián-Martínez et al., Phys. Rev. D 93, 122010 (2016)

[6] A. Albert et al., Phys. Rev. D 96, 022005 (2017)

[7] A. Albert et al., Eur. Phys. J. C 77, 911 (2017)

[8] A.D. Avrorin et al., arXiv:1810.10966 .

[9] A. Albert et al., ApJL L35, 850 (2017)

[10] S. Adrian-Martinez et al., JCAP 06, 008, (2013)

[11] M.G. Aartsen et al., Phys.Rev. D90 no.10, 102002 (2014)

[12] B. Baret for the ANTARES coll., Procedings of the Rencontres de Moriond: Gravitation (2015).

[13] A. Albert et al., ApJ 870 no.2, 134 (2019) 